Article DOI: https://doi.org/10.35219/ann-ugal-math-phys-mec.2019.1.07

\title{
CHEMOMETRIC APPLICATION OPERATING A PORTABLE LASER INFRARED SENSOR DETECTING ILLICIT PHENETHYLAMINES
}

\author{
Stefanut Ciochina ${ }^{1}$, Mirela Praisler ${ }^{2}$ \\ ${ }^{1}$ Faculty of Sciences and Environment, Department of Mathematics and Computer Science,,,Dunărea de Jos”, \\ University of Galati, Romania, e-mail: Stefanut.Ciochina@ugal.ro \\ ${ }^{2}$ Faculty of Sciences and Environment, Department of Chemistry, Physics and Environment,,,Dunărea de Jos” \\ University of Galati, Romania, e-mail: Mirela.Praisler@ugal.ro
}

\begin{abstract}
We are presenting a chemometrical application designed to recognize compounds having a molecular structure similar to the main controlled stimulant and hallucinogenic illicit phenetylamines, i.e. to amphetamines and their main precursors, the ephedrines. The input database contains their infrared laser spectra, which have been recorded with a new portable GC - IR sensor, in the spectral domain (1405 - $1150 \mathrm{~cm}^{-1}$ ), specific to its quantum cascade laser (QCL) source of electromagnetic radiation (UT8). A discriminating $\mathrm{w}_{\mathrm{TE}}$ feature weight was first determined by using the spectra of the targeted positives and the spectra of various compounds of forensic interest. The input database, formed with the $\mathrm{WTE}_{\mathrm{TE}}^{2}$ preprocessed laser infrared spectra, has been subjected to Principal Component Analysis (PCA). The scores plots have been analyzed in order to evaluate to what extent the amphetamines and their main precursors, the ephedrines, are forming specific and successfully distinguishable clusters. The results indicate that the application meets the requirements of forensic analytical tools.
\end{abstract}

Keywords: Amphetamines, ephedrines, chemometrics.

\section{INTRODUCTION}

The increasing occurrence of controlled stimulant and hallucinogenic illicit amphetamines on the black market has prompted many attempts to develop analytical instuments customized for their detection and chemometrical applications designed to automatically operate these sensors [1]. Moreover, as amphetamines are synthetic drugs, important efforts are also made for improving the efficiency of these new instruments from the point of view of the detection of their main precursors, the ephedrines [2,3].

The challenge is related to the fact that both these two classes of phenetylamines (amphetamines and ephedrines) have very small and very similar molecular structures. The highest similarity can be noticed especially between the class of stimulant amphetamines (analogues and homologues of amphetamine, $\alpha$-methylphenethylamine) and the class of ephedrines (ephedrine and its stereoisomers and diastereomers, e.g. norephedrine and pseudoephedrine) [1].

Hence, the detection instruments have to be very selective [3]. In this paper we are presenting the results obtained for the prototype of a portable GC-IR laser spectrometer that was developped for forensic purposes. The results indicate that the application successfully meets the requirements of forensic analytical tools. 


\section{EXPERIMENTAL PART}

The 36 spectra included in the database have been recorded in the spectral domain (1405 - 1150 $\mathrm{cm}^{-1}$ ), specific to its quantum cascade laser (QCL) source of electromagnetic radiation (UT8), with a resolution of $5 \mathrm{~cm}^{-1}[4,5]$. The database contains the spectra of 7 stimulant amphetamines (amphetamine and its main analogues and homologues, assigned class code M), 6 ephedrines (ephedrine and its main stereoisomers and diastereomers, assigned class code E), 6 hallucinogenic amphetamines (3,4methylenedioxyamphetamines and its main analogues, assigned class code T) and 17 negatives (nonphenethylamines, class code N) [6-8].

A discriminating $\mathrm{w}_{\mathrm{TE}}$ feature weight was first determined by using the spectra of the targeted positives and the spectra of various compounds of forensic interest [9]. The feature weight was determined by including the spectra of the hallucinogenic amphetamines $(\mathrm{T})$ and of the ephedrines $(\mathrm{E})$ in class I and the spectra of the stimulant amphetamines (M) and of the negatives (N) in class II [10-12].

The database formed with the $\mathrm{wTE}^{2}$ preprocessed laser infrared spectra, has been subjected to Principal Component Analysis (PCA) [13], by using the MATLAB software package. The scores plots have been analyzed in order to evaluate to what extent the amphetamines and their main precursors, the ephedrines, are forming specific and reliably distinguishable clusters [14]. These plots have been also been corroborated with the associated loading plots, in order to identify the absorptions that are the most important for the generation and discrimination of each cluster.

The minimum number of principal components necessary in order to perform a reliable class identity assignment of the modeled compounds has been then established by corroborating the findings of the PCA analysis with the results of a statistical analysis performed with the individual scores of PC1, $\mathrm{PC} 2$ and $\mathrm{PC} 3$.

\section{RESULTS AND DISCUSSION}

The $w_{T E}^{2}$ feature weight is presented in Fig. 1, which shows that $w_{T E}^{2}$ acts as a powerful selective amplifier: it increases significantly the intensity of the bands found around 1245 and $1190 \mathrm{~cm}^{-1}$, which are the most stable and the strongest absorptions of the hallucinogenic amphetamines $[6,7]$.

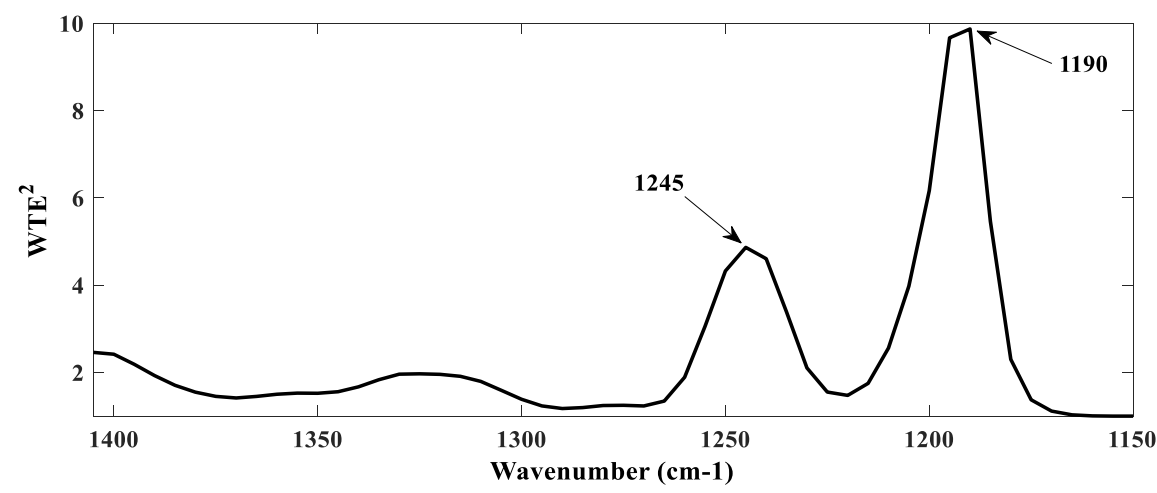

Fig. 1. wTE $^{2}$ selective amplifier used for processing the spectra before multivariate analysis

The $w_{T E}^{2}$ preprocessed spectra have been subjected to PCA. The analysis of the variance explained by each principal component (PC) indicated that the first three PCs are cumulating most of the explained variance (see Fig. 2). Hence, the score plots and the loading plots have been determined for the first three PCs. 


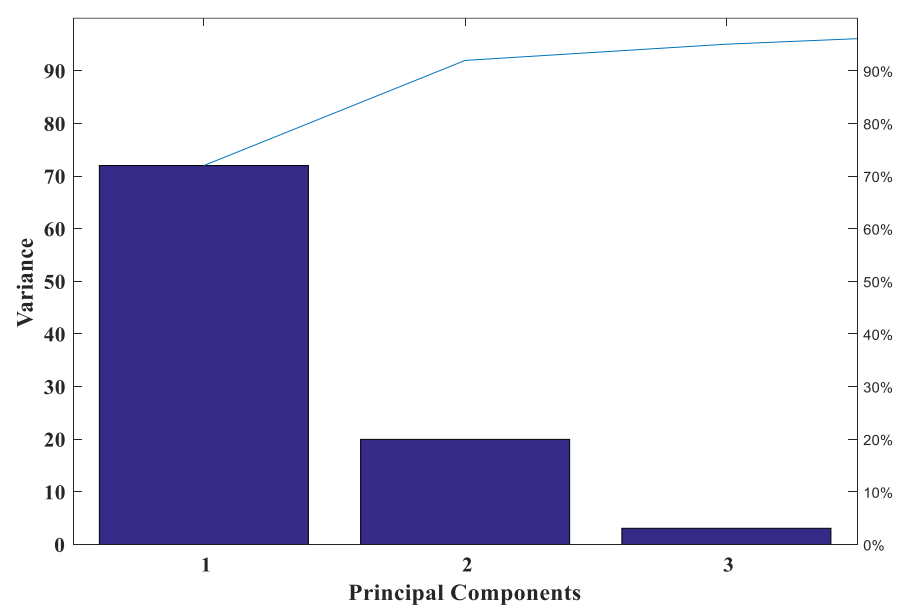

Fig. 2. Explained variance obtained with the $w T E^{2}$ preprocessed spectra

The score and loading plots obtained for PC1 and PC2 are presented in Fig. 3. The score plot (see Fig. 3a) indicates that the hallucinogenic amphetamines form the densest cluster. The loading plot (see Fig. 3b) shows that this remarkable density is due to the absorption band found around $1245 \mathrm{~cm}^{-1}$. The entire envelope of this band is essential for the formation of the $T$ cluster, as the wavenumbers contributing to the formation of this cluster range sequentially (with a $5 \mathrm{~cm}^{-1}$ resolution) between 1260 and $1230 \mathrm{~cm}^{-1}$. The ephedrines (E) form a more diffuse, but easily recognizable cluster, characterized by small positive PC1 scores and large negative PC2 scores (see Fig. 3a). This is mainly due to the absorptions they display between 1180 and $1210 \mathrm{~cm}^{-1}$ (see Fig. 3b). However, the classes of stimulant amphetamines $(\mathrm{M})$ and of negatives $(\mathrm{N})$ cannot be distinguished one from another based solely on their PC1 and PC2 scores, as these clusters overlap on a significant area found between the quadrants III and IV.

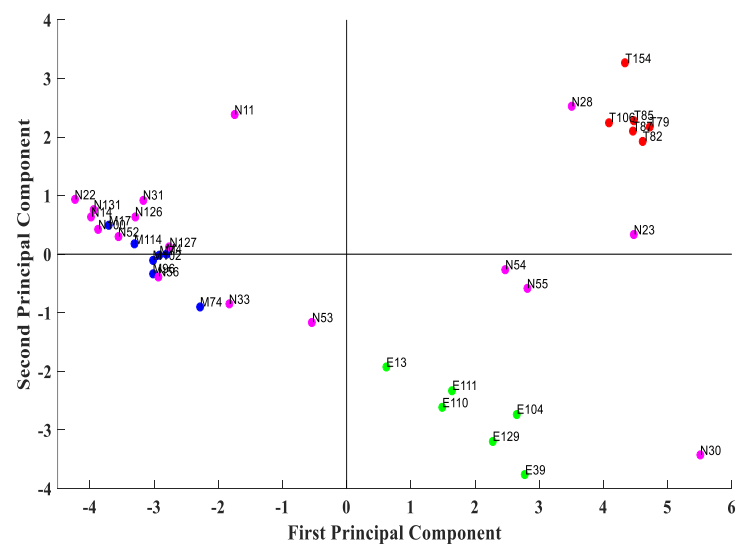

a)

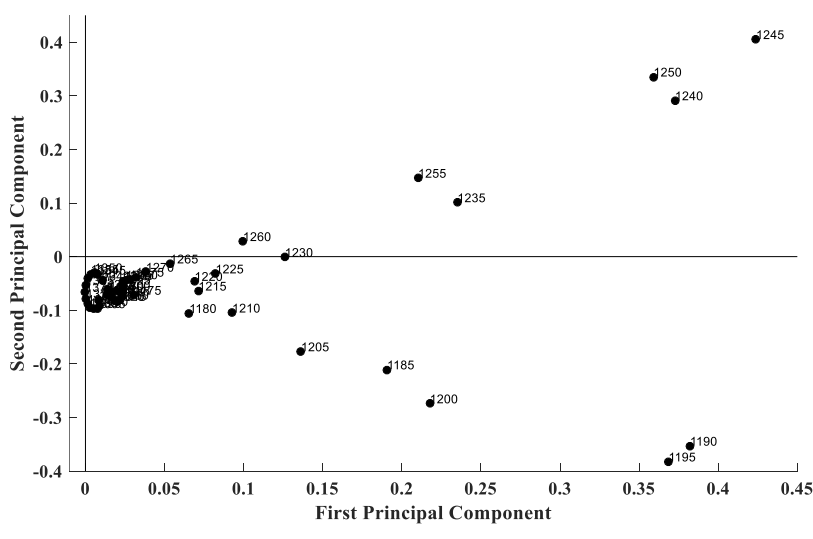

b)

Fig. 3. PC1 vs. PC2 plots obtained with the $w_{T E}^{2}$ processed spectra of stimulant amphetamines $(M)$, hallucinogenic amphetamines $(T)$ and negatives $(N)$ : a) score plot; $b)$ loading plot 
The same two clusters (E and T) may be clearly distinguished in the PC1 vs. PC3 score plot (see Fig. 4). Both clusters are located in quadrant IV, as opposed to the cloud formed by the $\mathrm{M}$ and $\mathrm{N}$ compounds, which is found at the border between quadrant III and quadrant IV. Hence, PC3 does not bring any improvement in the discrimination of the stimulant amphetamines from the negatives. This behavior is also reflected by the PC2 vs. PC3 plot (see Fig. 5): the hallucinogens, which are characterized by large positive PC2 scores, cluster in quadrant IV, while the ephedrines, which are characterized by large negative PC2 scores, form their cluster in quadrant III. The M and N substances cannot be distinguished, as the members of both these classes are characterized (with very few exceptions) by small (positive or negative) PC2 scores.

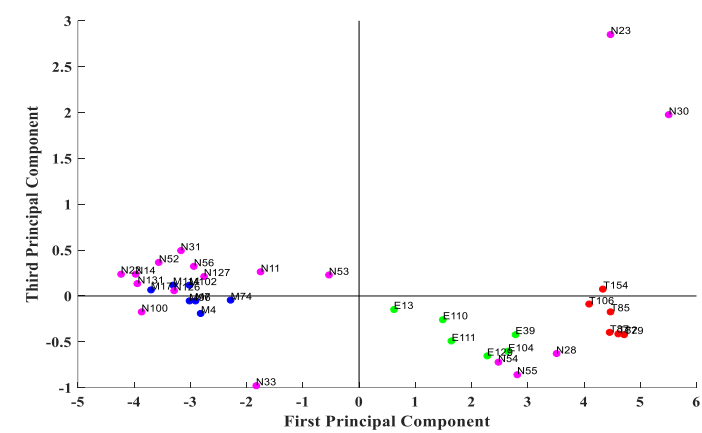

a)

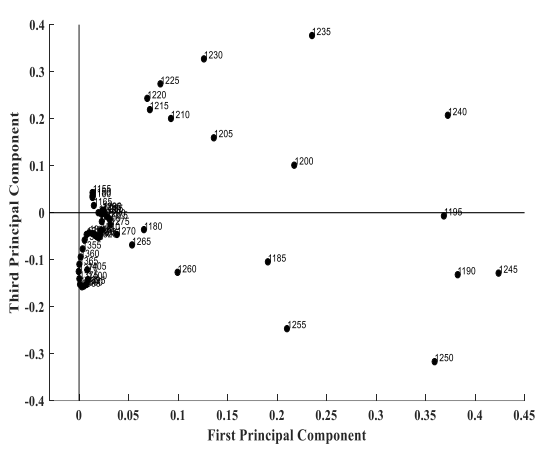

b)

Fig. 4. PC1 vs. PC3 plots obtained with the $w_{T}^{2}$ processed spectra of stimulant amphetamines $(M)$, hallucinogenic amphetamines $(T)$ and negatives $(N)$ : a) score plot; b) loading plot

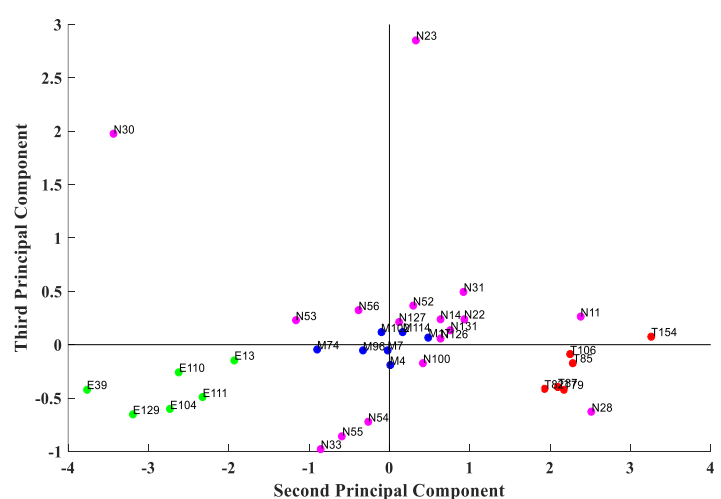

a)

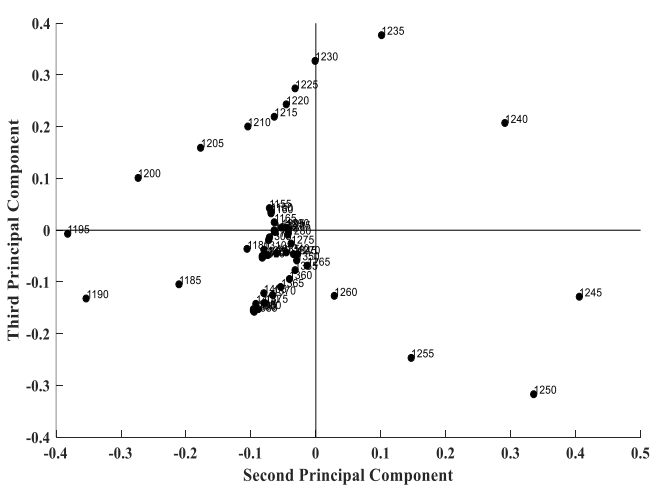

b)

Fig. 5. PC2 vs. PC3 plots obtained with the $w_{T E}^{2}$ processed spectra of stimulant amphetamines $(M)$, hallucinogenic amphetamines $(T)$ and negatives $(N)$ : a) score plot; $b$ ) loading plot

Hence, the analysis of the PCA plots indicates that: a) the $\mathrm{w}_{\mathrm{TE}}^{2}$ processed spectra can only be used for the (reliable) discrimination of $\mathrm{E}$ and $\mathrm{T}$ drugs of abuse from $\mathrm{M}$ and $\mathrm{N}$ compounds; $\mathrm{b}$ ) PC3 does not improve the discrimination that may be obtained with the PC1 and PC2 scores; c) the best discrimination between ephedrines (E) and hallucinogenic amphetamines (T) is ensured by the PC2 scores. 
These findings point out that the same classification results may be obtained with one (PC2) or a maximum of two PCs, i.e. PC1 and PC2. In order to make a final decision, we have performed a statistical analysis of the individual PC1, PC2 and PC3 scores (see Fig. 6). The results indicate that a reliable discrimination can be performed based on the scores of only one PC only in the case of the ephedrines. These precursors can be detected in a reliable way based only on their PC2 scores (see Fig. 6b). If the application is to be used for the simultaneous screening for $\mathrm{E}$ and $\mathrm{T}$ illicit drugs, then both PC1 and PC2 scores should be taken into account.

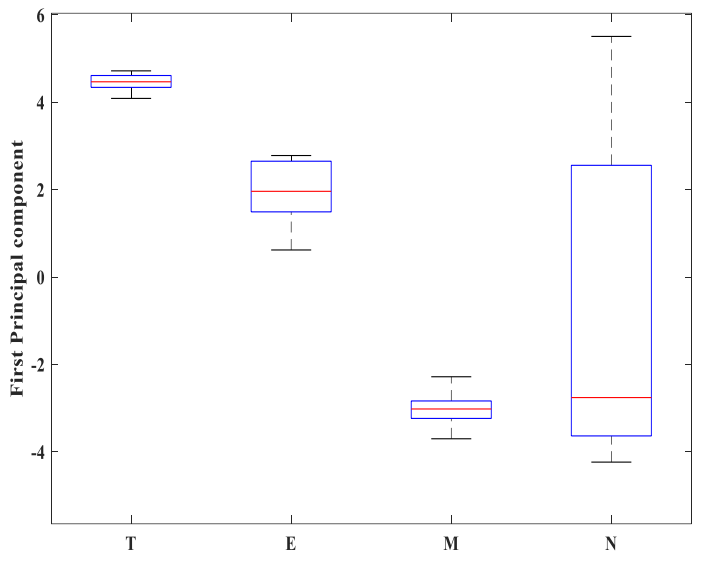

a)

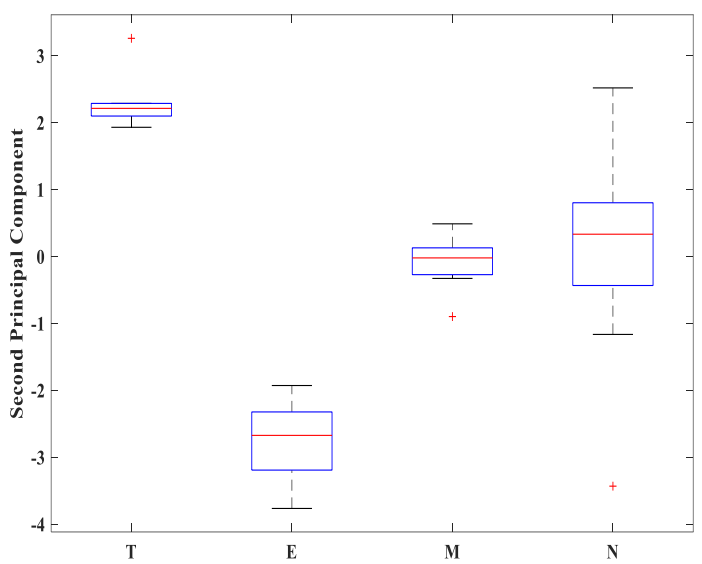

b)

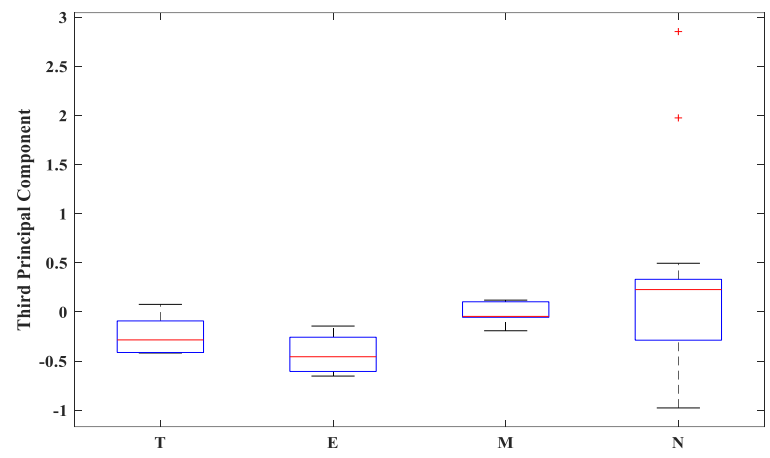

c)

Fig. 6. Box plot determined with the PCA scores of hallucinogenic amphetamines $(T)$, ephedrines (E), stimulant amphetamines $(M)$ and negatives $(N)$ : a) PC1; b) PC2; c) PC3

\section{CONCLUSIONS}

The findings presented in this study indicate that combining the PCA analysis with the statistical analysis of the individual PC scores is an efficient way to establish the minimum number of PCs needed for performing a reliable screening of given classes of compounds. In our case, the results indicate that the $\mathrm{WTE}_{\mathrm{TE}}^{2}$ processed spectra recorded with the new portable GC - IR sensor, in the $1405-1150 \mathrm{~cm}^{-1}$ spectral domain, can be successfully used for detecting ephedrines by analyzing the scores of only one PC, i.e. PC2. If the application is to be used as a multipurpose tool, i.e. to screen simultaneously for ephedrines and for hallucinogenic amphetamines, then the PC1 and PC2 scores should be taken into account. 


\section{Acknowledgements}

Part of the research has been funded by EC under the grant agreement $n^{\circ}$ FP7-SEC-2009-242309 DIRAC. The work of Stefanut Ciochina has been funded by the Romanian Ministry of European Funds within the POSDRU/107/1.5/S/76822 project. The authors are grateful for the financial support.

\section{References}

1. Karch S., Drug of Abuse Handbook, 2nd ed. CRC Press, Boca Raton, 2007.

2. Laing R. (Ed,), Hallucinogens. A Forensic Drug Handbook, Academic Press, London, 2003.

3. Chalmers J. M., Edwards H. G. M., Hargreaves M. D., Infrared and Raman Spectroscopy in Forensic science, Wiley, Chichester, 2012.

4. Praisler M., Ciochina S., Negoita C., Improved Selectivity in Detecting Controlled Amphetamines and their Main Precursors based on Laser Infrared Spectra, 2017 E-Health and Bioengineering Conference, EHB 2017, 28 July 2017, pp. 233-236. Article number 7995404.

5. Ciochina S., Praisler M., Negoita C., Cluster Analysis Evaluating the Automated Detection of Drugs of Abuse with a New Hollow Fiber based Quantum Cascade Laser Infrared Spectrometer, 2017 E-Health and Bioengineering Conference, EHB 2017, 28 July 2017, pp. 237-240. Article number 7995405.

6. Praisler M., Ciochina S., Coman M., Screening for Illicit Psychoactive Drugs Based on Pattern Recognition Methods, 5th International Symposium on Electrical and Electronics Engineering, ISEEE 2017, 20-22 October 2017, Galati, Romania

7. Ciochina S., Praisler M., Coman M., Hierarchical Cluster Analysis Applied for the Automated Recognition of Psychoactive Substances and of Their Main Precursors, $20175^{\text {th }}$ International Symposium on Electrical and Electronics Engineering (ISEEE), 20-22 October 2017, Galati, Romania.

8. Praisler M., Ciochina S., Coman M., Hunting for Illicit Psychoactive Substances and Precursors: a Multivariate Approach, $201721^{\text {st }}$ International Conference On System Theory, Control And Computing (ICSTCC), pp 248-253. Article number 8107042.

9. Ciochina S., Praisler M., Detecting Phenethylamines According To Their Pharmacological Activity, Annals Dunarea de Jos Univ. Galati, Fascicle II, Year XLI, 18, 2018.

10. Gosav S., Praisler M., Dorohoi D. O., Popa G., Structure - Activity Correlations for Illicit Amphetamines Using ANN and Constitutional Descriptors, Talanta 70, 922, 2006.

11. Gosav S., Praisler M., Dorohoi D. O., ANN Expert System Screening for Illicit Amphetamines using Molecular Descriptors, J. Mol. Struct. 834-836, 188, 2007.

12. Gosav S., Praisler M., Artificial Neural Networks Built for the Recognition of Illicit Amphetamines Using a Concatenated Database, Rom. Rep. Phys. 54 (9/10), 929, 2009.

13. Jolliffe I. T., Principal Component Analysis, $2^{\text {nd }}$ ed., Springer, New York, 2002.

14. Praisler M., Ciochina S., Global clustering quality coefficient assessing the efficiency of PCA class identity assignment, J. Anal. Methods Chem., 2014, Article ID 342497, 2014. 